\title{
SARS-COV-2 EN LATINOAMÉRICA DESDE LA PERSPECTIVA DEL MÉDICO FAMILIAR
}

\section{SARS-COV-2 IN LATIN AMERICA FROM THE PERSPECTIVE OF FAMILY DOCTORS}

\author{
Rossana D’Addosio-Valera ${ }^{1,2 *}$, Carla P. Navarro-Márquez ${ }^{3}$, José M. Ramírez-Aranda y G Gloria Córdova-Currea ${ }^{5}$ \\ 'Centro Clínico de Medicina Familiar Indio Mara, Instituto de Enfermedades Cardio-Vasculares, Estado Zulia, Maracaibo, Venezuela; \\ 2Departamento de Salud Pública, Universidad del Zulia, Estado Zulia, Maracaibo, Venezuela; ${ }^{3}$ Facultad de Medicina, Instituto Endocrino \\ Metabólico, Universidad del Zulia, Estado Zulia, Maracaibo, Venezuela; ${ }^{4}$ Departamento de Medicina Familiar, Universidad Autónoma de Nuevo \\ León, Monterrey, Nuevo León, México; ${ }^{5}$ nstituto de Salud Pública, Universidad de Copenhague, Copenhague, Dinamarca
}

RESUMEN: La pandemia ha sido manejada de forma diferente por la atención primaria, con poca evidencia publicada. Objetivo: Conocer la percepción del rol del médico familiar dentro de la pandemia de enfermedad por coronavirus 2019 (COVID-19) en Latinoamérica. Material y método: Estudio observacional, descriptivo y transversal, donde se aplicó un cuestionario electrónico autoadministrado y distribuido por medio de asociaciones de profesionales de atención primaria de varios países en una muestra por conveniencia. Se usó la aceptación del cuestionario como el consentimiento del estudio. Resultados: Los países con mayor porcentaje de respuesta fueron México (29.9\%), Chile (16.2\%) y Venezuela (15.6\%). La mayoría reportó haber atendido pacientes con diagnóstico sospechoso o confirmado de COVID-19 (73.4\%), de los cuales el 70\% consideró que no existió suficiente dotación de equipos de protección personal para afrontar la crisis y el $52.6 \%$ señaló que el personal de salud no estaba debidamente entrenado. Asimismo, el 62.3\% de los participantes consideró que existió prevalencia de los intereses políticos sobre los epidemiológicos, mientras que el $71 \%$ percibió deficiencias en la vinculación de la agenda política/económica con la agenda epidemiológica. Conclusiones: La forma de afrontar la pandemia de los países latinoamericanos fue homogénea, con un enfoque hospitalario, relegando a la atención primaria en salud, con escasa participación de los médicos de familia.

Palabras clave: COVID-19. Coronavirus. SAR- CoV-2. Medicina Familiar. Estrategias. Latinoamérica.
ABSTRACT: The pandemic has been managed differently by primary care with little published evidence Objective: To know the perception of the role of the family doctor within the coronavirus disease 2019 (COVID-19) pandemic in Latin America. Material and method: An observational, descriptive, and cross-sectional study, where a self-administered electronic questionnaire distributed through primary care networks from several countries was conducted. Convenience sampling was applied. The acceptance of the questionnaire was used as the consent of the study. Results: The countries with the highest response percentage were Mexico (29.9\%), Chile (16.2\%) and Venezuela (15.6\%). The majority of participants reported having treated patients with a suspected or confirmed diagnosis of COVID-19 (73.4\%), of which $70 \%$ considered that there was not enough provision of personal protective equipment to face the crisis, and $52.6 \%$ indicated that the health personnel were not properly trained. Likewise, $62.3 \%$ of the participants considered that there was a prevalence of political over epidemiological interests, while $71 \%$ perceived deficiencies in linking the political / economic agenda with the epidemiological agenda. Conclusions: The way of facing the pandemic in Latin American countries was homogeneous, with a clearly hospital approach relegating PHC with little participation from Family Physicians.

Key words: COVID-19. Coronavirus. SAR- CoV-2. Family Medicine. Strategies. Latin America.
Correspondencia:

*Rossana D'Addosio-Valera

E-mail: rodava2@gmail.com
Fecha de recepción: $30-12-2020$

Fecha de aceptación: 22-03-2021
Disponible en internet: 05-05-2021

Rev Mex Med Fam. 2021;8:7-14 DOI: 10.24875/RMF.20000217

2007-9710 / @ 2021 Federación Mexicana de Especialistas y Residentes en Medicina Familiar. Publicado por Permanyer. Este es un artículo open access bajo la licencia CC BY-NC-ND (http://creativecommons.org/licenses/by-nc-nd/4.0/). 


\section{INTRODUCCIÓN}

Similar a la experiencia vivida en epidemias anteriores, el tratamiento para la infección por el coronavirus 2 del síndrome respiratorio agudo grave (SARS-CoV-2) es un reto al que se enfrentan los diversos sistemas de salud del mundo, donde las medidas de prevención y diagnóstico oportuno continúan siendo la piedra angular ${ }^{1}$, ámbito en el que la atención primaria y sus principales efectores, los médicos familiares (MF), desempeñan un papel fundamental ${ }^{2}$. Pese a esto, la Sociedad Española de Medicina Familiar y Comunitaria (semFYC) declaró que inicialmente se gestionó la atención de la pandemia a espaldas de la atención primaria en salud (APS), manejando los servicios bajo un enfoque hospitalario y trasladando los recursos de salud ambulatoria a hospitales improvisados ${ }^{3}$, poniendo en evidencia las debilidades y déficits crónicos que padece la APS.

Pese a ser el primer eslabón en la atención de los pacientes, al especialista en medicina familiar se le limitó de pruebas diagnósticas e incluso de equipos de protección personal (EPP) suficientes durante las primeras fases de la pandemia, poniéndolos en situación de vulnerabilidad y limitando el proceso de toma de decisiones ${ }^{4}$. Poco valieron las advertencias de los galenos españoles y en Latinoamérica replicamos sus mismos errores ${ }^{5}$, lo cual terminó por ocasionar el colapso del sistema y el aumento de la propagación del virus ${ }^{1}$.

En la búsqueda de soluciones, esta crisis ha impulsado la transformación de la medicina presencial a un ambiente virtual, siendo pionera la medicina familiar desde la APS, con la propuesta de la práctica de telemedicina con asistencia remota a pacientes en su domicilio $^{6}$. Sin embargo, existen muy pocos estudios que evidencien cuál ha sido el papel del MF latinoamericano durante la pandemia, por lo que el objetivo de este estudio es conocer la percepción del rol del médico familiar dentro de la pandemia de enfermedad por coronavirus 2019 (COVID-19) en Latinoamérica.

\section{MATERIAL Y MÉTODO}

Estudio observacional, descriptivo y transversal, donde la población de estudio fueron médicos de atención primaria, especialistas o residentes en medicina familiar en Latinoamérica. Para determinar el tamaño muestral se aplicó la fórmula para el cálculo de muestras con poblaciones infinitas ${ }^{7}$ asignando un intervalo de confianza del $95 \%$ y un margen de error del $5 \%$, obteniéndose un tamaño muestral de 384 individuos. Sin embargo, al tratarse de un estudio no probabilístico, realizado a gran escala en un contexto de pandemia, se consideró aceptable fijar un margen de error del $8 \%$ para un tamaño muestral de 150 individuos. Los individuos fueron escogidos mediante un muestreo no aleatorio por conveniencia.

Se elaboró un cuestionario electrónico ad hoc, que fue validado por tres expertos cuyas observaciones fueron incorporadas en la versión final. El cuestionario constó de tres partes que agruparon las variables de estudio. La primera referente a variables epidemiológicas relacionadas con el SARSCoV-2/COVID-19, con cinco preguntas de selección: primer caso confirmado, cumplimiento de las medidas sanitarias (escala de Likert: muy deficiente, deficiente, aceptable y adecuado), tipo de vigilancia epidemiológica y fase de la pandemia. La segunda parte referente a las competencias del MF, con nueve preguntas de selección: lugar de trabajo, actividad como MF, manejo de pacientes con COVID-19, forma de adquisición de EPP, nivel de liderazgo en la toma de decisiones (escala del 1 al 5), uso de telemedicina, hallazgos revelados sobre el sistema de salud, participación y reconocimiento de la medicina familiar (escala de Likert: en desacuerdo, parcialmente de acuerdo y totalmente de acuerdo), y probables medidas exitosas no consideradas.

Se realizó prueba piloto para evaluar las cualidades psicométricas del instrumento, tiempo de llenado y pertinencia. El análisis de confiabilidad de las dos preguntas con escalas tipo Likert reveló una $a$ de Cronbach de 0.870, indicando consistencia interna muy buena y aceptable para la sección de «Cumplimiento de las medidas sanitarias ante la pandemia», y para la sección de «Participación y reconocimiento de la medicina familiar» alcanzó un 0.634 , indicando consistencia interna medianamente aceptable. 
Tabla 1. Percepción del cumplimiento de medidas sanitarias implementadas para la atención de casos de COVID-19. LATAM, $2020(n=154)$

\begin{tabular}{|l|c|c|}
\hline Variable & $\begin{array}{c}\text { Deficiente } \\
\mathbf{n}(\%)\end{array}$ & $\begin{array}{c}\text { Adecuada } \\
\mathbf{n}(\%)\end{array}$ \\
\hline La cuarentena cumplió sus objetivos & $83(53.9)$ & $71(46.1)$ \\
\hline La comunidad cumplió las normas: uso de mascarilla, distanciamiento social, cuarentena & $101(65.6)$ & $53(34.4)$ \\
\hline $\begin{array}{l}\text { Existe dotación de equipos médicos suficiente para afrontar los casos: camas de hospital, } \\
\text { ventiladores, medicamentos }\end{array}$ & $103(66.9)$ & $51(33.1)$ \\
\hline El personal de salud estaba debidamente entrenado para atender los casos y/o derivarlos & $81(52.6)$ & $73(47.4)$ \\
\hline La agenda política/económica estaba vinculada con la agenda epidemiológica & $110(71.4)$ & $44(28.6)$ \\
\hline $\begin{array}{l}\text { La comunidad fue creativa, organizada con apoyo espontáneo (p. ej., manufactura de tapabocas/ } \\
\text { mascarillas caseras, esquema de horario para uso de áreas comunes, etc.) }\end{array}$ & $82(53.2)$ & $72(46.8)$ \\
\hline
\end{tabular}

El cuestionario fue difundido por correo electrónico y redes sociales (Facebook y WhatsApp). Aceptados los términos del consentimiento informado se desplegaron las preguntas. Se contactó con los participantes por medio de los líderes regionales latinoamericanos, con información sobre el objetivo del estudio. De aceptar, se les pidió difundir la encuesta creando efecto «bola de nieve». La encuesta fue autoaplicada, anónima y voluntaria por medio de la plataforma de Google Forms (www.docs.google.com/ forms), disponible del 24 de agosto al 1 de septiembre. Se excluyeron las encuestas de la prueba piloto. Se consideró criterio de inclusión formularios con datos en más del $90 \%$.

Los datos, registrados en Excel, se exportaron al programa SPSS versión 18.0 con datos verificados para reducir sesgos de transcripción. Se realizó análisis descriptivo univariante y bivariante. Las variables cuantitativas continuas se expresan en medidas de tendencia central y dispersión. Las variables cualitativas con frecuencias absolutas y relativas en porcentajes.

Para evidenciar un análisis estadístico cualitativo consistente, las respuestas correspondientes a la sección de "Cumplimiento de las medidas sanitarias ante la pandemia» fueron recodificadas a dicotómicas (deficiente y adecuado). Adicionalmente, para el análisis cuantitativo se sumaron las respuestas de todos los ítems en cada una de las dos secciones del instrumento, cuyo total fue expresado en media \pm desviación estándar
(DE) comparado entre los países participantes, con test Anova de un factor y significancia estadística de $\mathrm{p}<0.001$.

\section{RESULTADOS}

Un total de 155 MF participaron en el estudio, de los cuales uno rechazó el consentimiento informado y fue excluido del análisis. Los países con mayor porcentaje de respuesta fueron México (29.9\%), Chile (16.2\%) y Venezuela (15.6\%), seguidos en menor frecuencia por Ecuador (7.8\%), Panamá y Colombia (5.2\% cada uno), y otros con participaciones mucho menor es incluyen Argentina y Bolivia ( $3.2 \%$ cada uno), Costa Rica (4.5\%), Guatemala (1.3\%), Paraguay $(2.6 \%)$, Perú y Puerto Rico (1.9\% cada uno), República Dominicana y Uruguay ( $0.6 \%$ cada uno). Para el momento del estudio, la mayoría de los países se encontraba en fase generalizada de la pandemia (71.4\%).

Al evaluar el cumplimiento de las medidas sanitarias implementadas (Tabla 1), la mayoría de los encuestados reportó que la cuarentena no cumplió con sus objetivos de forma adecuada, y consideró que el cumplimiento de las normas por parte de la comunidad fue deficiente. Alrededor del $70 \%$ piensa que tanto la dotación de equipos médicos como la vinculación de la agenda política/económica con la agenda epidemiológica fue deficiente. Por otro lado, un poco más de la mitad de los entrevistados señaló que el personal de salud no estaba debidamente entrenado para atender casos o 
Tabla 2. Forma de adquisición del equipo de protección personal según país de procedencia en los tres países con mayor tasa de respuesta

\begin{tabular}{|l|c|c|c|c|}
\hline Variable & $\begin{array}{c}\text { Chile } \\
\mathbf{n}(\%)\end{array}$ & $\begin{array}{c}\text { México } \\
\mathbf{n}(\%)\end{array}$ & $\begin{array}{c}\text { Venezuela } \\
\mathbf{n}(\%)\end{array}$ & $\begin{array}{c}\text { Otros* } \\
\mathbf{n}(\%)\end{array}$ \\
\hline Comprado de forma personal & $2(8.0)$ & $18(39.1)$ & $10(41.7)$ & $14(23.7)$ \\
\hline Otorgado por la institución donde labora & $12(48.0)$ & $2(4.3)$ & $4(16.7)$ & $21(35.6)$ \\
\hline Mixto, parte autoadquirido, parte dado por la institución & $11(44.0)$ & $26(56.5)$ & $10(41.7)$ & $24(40.7)$ \\
\hline
\end{tabular}

*Argentina, Bolivia, Colombia, Costa rica, Ecuador, Guatemala, Panamá, Paraguay, Perú, Puerto Rico, República Dominicana y Uruguay.

Tabla 3. Percepción del rol de medicina familiar en el sistema de salud en los países estudiados durante la pandemia de COVID-19. LATAM, 2020

\begin{tabular}{|l|c|c|c|}
\hline Variable & $\begin{array}{c}\text { En desacuerdo } \\
\mathbf{n}(\%)\end{array}$ & $\begin{array}{c}\text { Parcialmente de acuerdo } \\
\mathbf{n}(\%)\end{array}$ & $\begin{array}{c}\text { Totalmente de acuerdo } \\
\mathbf{n}(\%)\end{array}$ \\
\hline La red de atención primaria es escasa o inexistente & $45(29.3)$ & $82(53.2)$ & $76(17.5)$ \\
\hline $\begin{array}{l}\text { El nivel hospitalario soportó todo el peso de la } \\
\text { atención de pacientes }\end{array}$ & $45(29.2)$ & $72(46.4)$ & $60(39.0)$ \\
\hline $\begin{array}{l}\text { La capacidad resolutiva de la medicina familiar no } \\
\text { fue tomada en cuenta }\end{array}$ & $22(14.3)$ & $57(37.0)$ & $69(44.8)$ \\
\hline $\begin{array}{l}\text { La medicina familiar obtuvo reconocimiento } \\
\text { en el sistema de salud }\end{array}$ & $28(18.2)$ & $70(45.5)$ & $67(43.5)$ \\
\hline $\begin{array}{l}\text { Participación de medicina familiar en la } \\
\text { formulación de políticas de salud }\end{array}$ & $17(11.0)$ & $93(60.4)$ & $35(22.7)$ \\
\hline $\begin{array}{l}\text { Se lograron alianzas con otras asociaciones } \\
\text { involucradas en el proceso de toma de decisiones }\end{array}$ & $26(16.9)$ & & \\
\hline
\end{tabular}

derivarlos, igualmente señalan que la organización de la comunidad no era adecuada.

Respecto a la forma de adquisición de los EPP, los médicos chilenos reportaron frecuentemente que fue otorgado por la institución laboral (48.0\%), para los mexicanos fue mixta, mientras que los venezolanos lo adquieren por su propio pecunio (Tabla 2).

$\mathrm{Al}$ evaluar el rol del MF durante la pandemia, el 55.2\% ( $\mathrm{n}=85)$ de los encuestados reportó trabajar en centros de salud públicos, de forma asistencial el $82.5 \%(n=127)$, con nivel de liderazgo en la toma de decisiones con alto porcentaje de atención de pacientes con diagnóstico sospechoso o confirmado de COVID-19 (73.4\%).

Respecto a la participación y reconocimiento de la medicina familiar en el sistema de salud, en la tabla 3 se puede observar que la mayoría consideró estar parcialmente de acuerdo en que la red de APS es escasa o inexistente, el nivel hospitalario soportó todo el peso de la atención de pacientes, la participación de la medicina familiar en la formulación de políticas de salud fue parcial, así como las alianzas logradas con otras asociaciones involucradas en la toma de decisiones. Asimismo, el 39.0\% estuvo totalmente de acuerdo en que la capacidad resolutiva de la medicina familiar no fue tomada en cuenta, pese a que, en contraste, el $44.8 \%$ percibió que obtuvo adecuado reconocimiento.

El análisis cuantitativo de los dos aspectos principales evaluados en este estudio se pueden observar en la tabla 4 , evidenciando que la media de la percepción de los encuestados sobre las medidas sanitarias implementadas por las autoridades políticas y epidemiológicas y su cumplimiento por parte de la población fue mayor en Costa Rica (17.8 \pm 5.1), seguido por Colombia (17.2 \pm $3.7)$ y Argentina $(16.4 \pm 2.7)$, mientras que Venezuela $(10.9 \pm 2.8)$ y Bolivia (10.8 \pm 2.6) exhibieron las menores medias, siendo estas diferencias estadísticamente significativas. Por otra parte, la percepción de la 
Tabla 4. Media de la percepción de los médicos familiares sobre cumplimiento de las medidas sanitarias implementadas y la participación de la medicina familiar durante la pandemia de COVID-19. LATAM, 2020

\begin{tabular}{|l|c|c|c|c|}
\hline Variable & \multicolumn{2}{|c|}{$\begin{array}{c}\text { Cumplimiento de la medidas sanitarias } \\
\text { implementadas* }\end{array}$} & \multicolumn{2}{c|}{$\begin{array}{c}\text { Participación de la medicina familiar } \\
\text { en la pandemia* }\end{array}$} \\
\hline País & Media & DE $( \pm)$ & 14.0 & DE $( \pm)$ \\
\hline Argentina & 16.4 & 2.7 & 12.6 & 1.0 \\
\hline Bolivia & 10.8 & 2.7 & 11.9 & 2.4 \\
\hline Chile & 16.3 & 3.4 & 13.0 & 2.4 \\
\hline Colombia & 17.3 & 3.7 & 10.4 & 2.0 \\
\hline Costa Rica & 17.9 & 5.1 & 14.3 & 2.1 \\
\hline Ecuador & 12.8 & 2.2 & 12.2 & 1.9 \\
\hline México & 11.2 & 3.4 & 12.4 & 2.2 \\
\hline Panamá & 16.0 & 2.3 & 13.9 & 1.9 \\
\hline Venezuela & 10.9 & 2.8 & 13.0 & 2.3 \\
\hline Otros & 14.7 & 2.6 & & 2.2 \\
\hline
\end{tabular}

*Diferencias estadísticamente significativas $(p<0.001)$ evaluadas mediante el test Anova de un factor. ${ }^{\dagger}$ Otros: Guatemala, Paraguay, Perú, Puerto Rico, República Dominicana y Uruguay. DE: desviación estándar.

participación y reconocimiento de la medicina familiar como especialidad reflejó resultados homogéneos, con una media mayor en Ecuador (14.2 \pm 1.9$)$ y Argentina $(14 \pm 1)$ y menor en Costa Rica (10.4 \pm 2$)$, siendo diferencias estadísticamente significativas.

Respecto a las nuevas formas de servicio de salud virtuales emergentes, el $46.1 \%$ de los MF reveló haber incorporado en su rutina profesional la educación médica virtual, como administrador, facilitador o participante. Un poco más de un tercio señaló haber incorporado el uso de redes sociales como medio de promoción (Facebook, IG, Tik-Tok), mientras que solo el 5.2\% reportó el uso de consultorio virtual o telemedicina. Finalmente, al indagar sobre las lecciones y desafíos para la medicina familiar aprendidas como especialidad, el $62.3 \%$ consideró que el sistema de salud reveló preeminencia de los intereses políticos sobre los epidemiológicos. Cuatro de cada 10 entrevistados señaló que hubiese sido exitoso establecer un sistema de notificación homogéneo en la región que facilite comparar los datos entre países.

\section{DISCUSIÓN}

En Latinoamérica la debilidad de los sistemas de protección social y de salud han exacerbado la vulnerabilidad de la región ante la pandemia, afectando el cumplimiento de las medidas de distanciamiento físico necesarias para controlar la transmisión por parte de la comunidad ${ }^{8}$. Esto último se evidencia en nuestro estudio, donde el $65.6 \%$ de los encuestados consideró que el cumplimiento de las normas por parte de la comunidad fue deficiente.

La medicina familiar posee las mejores cualidades para responder de forma adecuada ante esta crisis de salud ${ }^{9}$. De hecho, países como Singapur, Hong Kong y Corea del Sur lograron controlar la pandemia en sus primeras fases de forma aceptable al contar con una adecuada red de atención primaria $^{10-12}$. Sin embargo, nuestro estudio reveló que en la mayoría de los países latinoamericanos optó por un enfoque hospitalocentrista, al igual que en España, China o Italia ${ }^{3}$, sin tomar en cuenta la capacidad resolutiva de estos profesionales y dejando en evidencia una red de APS escasa o inexistente. Esto último entra en conflicto con el hecho de que se estima que alrededor del 80\% de los casos leves y moderados buscan los servicios de APS como punto de entrada para obtener atención médica ${ }^{13,14}$. Investigaciones realizadas en Canadá apuntan que, en etapas tempranas de la pandemia, tres cuartas partes (73\%) de los MF se 
encontraban evaluando pacientes para detectar COVID-1915, un hallazgo que coincide con el alto porcentaje de atención de pacientes con diagnóstico sospechoso o confirmado de COVID-19 que expresaron nuestros médicos (73.4\%).

La principal demanda mundial del personal de salud frente a la COVID-19 es la dotación de EPP, como lo reportan estudios realizados en Canadá y Pakistán, donde el 55 y el $92 \%$ de los MF encuestados señalan el problema ${ }^{15,16}$. De forma similar, según el estudio de Ortiz, et al. en Argentina la dimensión percibida como inadecuada fue la falta de recursos institucionales y de $\mathrm{EPP}^{17}$, en concordancia con nuestros hallazgos, que revelan que no existe suficiente dotación de equipos médicos para afrontar los casos, obligando a un porcentaje importante de los médicos a adquirir su EPP independientemente (28.6\%).

Otro de los hallazgos que es importante destacar es que un elevado porcentaje de nuestros médicos (52.6\%) señaló que el personal de salud no estaba debidamente entrenado para atender los casos de COVID-19 y/o derivarlos, al igual que el estudio argentino, que expone la necesidad de mayor capacitación sobre cómo lidiar con la enfermedad $^{17}$, lo cual pone de manifiesto la necesidad de capacitación del personal, así como la alta demanda de lineamientos unificados.

La respuesta ante la pandemia revela la ausencia de orientaciones estratégicas a nivel global respecto a las competencias y alcances de la atención primaria, algo que se arrastra desde hace décadas ${ }^{18}$. De hecho, revisiones tan antiguas de la literatura como las referentes a la pandemia de influenza de 1918 ya señalan interferencias en la participación de los médicos de atención primaria por falta de apoyo gubernamental y disponibilidad de recursos ${ }^{19}$. En el estudio de Yu, et al. en Hong Kong, el 85\% de los encuestados sugirieron que el gobierno y/o las autoridades de salud locales deberían instituir medidas para facilitar que los MF de primera línea respondan al brote de COVID- $19^{20}$. Asimismo, al indagar sobre las lecciones y desafíos para la medicina familiar aprendidas como especialidad en nuestro estudio, el $62.3 \%$ de los participantes consideró que el sistema de salud prefería intereses políticos sobre los epidemiológicos, mientras que el $71 \%$ percibió deficiencias en la vinculación de la agenda política/económica con la epidemiológica.

De esta forma, la pandemia ha demostrado la importante interdependencia que debe existir entre la salud y la economía ${ }^{8}$. Los sistemas de salud de los países de la región tienen importantes debilidades en la asignación de recursos al primer nivel de atención, estando por debajo de los parámetros recomendados del 30\% del gasto público $^{8}$. Si bien los hospitales se encuentran bajo enorme presión durante esta pandemia, la evidencia científica demuestra que invertir preferentemente en estos, lejos de disminuir la mortalidad, la aumenta ${ }^{18}$, al contrario del efecto que supone invertir en APS, si se tiene en cuenta que las medidas rápidas para contener y mitigar la pandemia dependen de la identificación y aislamiento temprano de los casos, reduciendo la carga hospitalaria ${ }^{12}$. Así pues, para resolver la crisis institucional y sanitaria derivada de la COVID-19, en concordancia con lo señalado en el 2005 por Starfield, et al. respecto a la meta de lograr una distribución más equitativa de la salud en las poblacio$n^{n} s^{21}$, se debe lograr un financiamiento adecuado para la AP, abarcando tres ámbitos: inversión en recursos humanos, en tecnología e infraestructuras ${ }^{18}$.

Otra de las exigencias que han emergido durante esta crisis es la incorporación de nuevas tecnologías en la comunicación entre profesionales y pacientes ${ }^{22}$, donde los MF han figurado como pioneros en el seguimiento telemático de los pacientes con diagnóstico de COVID-19, reduciendo los desplazamientos a los centros de salud ${ }^{23}$. La Academia Estadounidense de Médicos de Familia (AAFP) apoya el uso ampliado de la telemedicina como herramienta importante y eficiente para ayudar a cuidar a los pacientes a medida que la pandemia avanza ${ }^{24}$. Sin embargo, las tasas mundiales de utilización de servicios remotos siguen siendo bajas como consecuencia de la incertidumbre legal, los problemas de pago, los desafíos técnicos y la falta de familiaridad del paciente y el médico ${ }^{9,25}$. Respecto a esto último, un 
porcentaje importante de nuestros encuestados reveló haber incorporado en su rutina profesional la educación médica virtual, así como el uso de redes sociales como medio de promoción de salud, mientras que un bajo porcentaje (5.2\%) reportó el uso de consultorio virtual o telemedicina.

En efecto, este momento representa una gran oportunidad para evaluar la relación riesgo-beneficio para la atención médica virtual no solo en el contexto de COVID-19, sino como nueva alternativa en el seguimiento y control de pacientes con enfermedades crónicas ${ }^{23}$. Por lo tanto, es necesario proporcionar directrices y recomendaciones para educar a los médicos y pacientes, por medio del establecimiento de modelos y guías para el uso de telemedicina, tales como la Guía de Buenas Prácticas y Recomendaciones para el uso de Telemedicina durante la epidemia de COVID-19 en Chile ${ }^{26}$, o la hoja informativa sobre COVID-19 titulada Teleconsulta durante una Pandemia, desarrollada por la Organización Panamericana de la Salud ${ }^{27}$.

\section{LIMITACIONES}

A pesar de contactar con los líderes locales de cada país y difundir la encuesta por las redes sociales, la respuesta de los participantes fue escasa, afectando la tasa de cobertura estimada y por ende la precisión de los resultados, sin embargo, se consideró que la información era muy interesante, al reflejar la percepción de los MF en un momento donde la incertidumbre parecía dominar el escenario. No hubo representación de todos los países de Latinoamérica, quizás porque el formulario estuvo disponible por poco tiempo, así que generalizar los resultados no es viable. Los que respondieron la encuesta pudieron tener mayor accesibilidad al cuestionario electrónico por encontrase en áreas céntricas y quizás mejor dotadas, lo que representa un punto de vista diferente al de zonas aisladas o selváticas, lo que representa un probable sesgo de percepción. Los participantes fueron autoseleccionados, aun así, se prefirió un muestreo de voluntarios por ser más factible y viable al muestreo aleatorio. Los datos fueron recogidos durante un momento de la historia de la pandemia de probable agotamiento informático, lo que pudo afectar su alcance.

\section{Aportaciones del estudio}

- Los MF refrendan su compromiso de atención a la población, a pesar de la falta de insumos.

- Las políticas de salud siguen enfocadas en un modelo hospitalario.

- La APS es un modelo flexible que se adapta a los cambios y usa las herramientas tecnológicas a su favor para el beneficio de sus pacientes, las familias y la comunidad.

\section{CONCLUSIÓN}

A pesar de la heterogeneidad que muestran los países latinoamericanos, la forma de afrontar la pandemia fue similar, con un enfoque netamente hospitalario relegando a la APS a un segundo nivel, con escasa participación de los MF. La prevalencia de los intereses políticos sobre los epidemiológicos fue una constante entre los países estudiados, donde la disponibilidad de equipos de protección personal figuró como una preocupación importante entre los encuestados. Finalmente, las herramientas tecnológicas como la telemedicina y la teleeducación se presentaron como recursos emergentes que muy probablemente van a coexistir con la atención presencial.

\section{FINANCIAMIENTO}

Para la realización de este estudio no se recibió financiamiento externo.

\section{CONFLICTO DE INTERESES}

Los autores declaramos no tener conflicto de intereses.

\section{RESPONSABILIDADESÉTICAS}

Protección de personas y animales. Los autores declaran que para esta investigación no se han realizado experimentos en seres humanos ni en animales.

Confidencialidad de los datos. Los autores declaran que han seguido los protocolos de 
su centro de trabajo sobre la publicación de datos de pacientes.

Derecho a la privacidad y consentimiento informado. Los autores declaran que en este artículo no aparecen datos de pacientes.

\section{BIBLIOGRAFÍA}

1. Palomino V, Cahuina-Lope P. La medicina familiar y comunitaria en la pandemia por COVID-19: Contribuciones y desafíos. Health Care \& Global Health. 2020;4(1):24-7.

2. Varela Rueda $C E$, Reyes Morales $H$, Albavera Hernández $C$ Ochoa Díaz-López H, Gómez Dantés H, García Peña C. La medicina familiar en México: presente y futuro. Gac Méd Méx 2016;152(1):135-40.

3. Iparraguirre ST, Álvarez RM. La semFYC y la medicina de familia en tiempos del coronavirus. Aten Primaria. 2020;52(5):291-3.

4. Llisterri Caro JL. La maldita pandemia: una oportunidad para la Atención Primaria de Salud. SEMERGEN. 2020:46(3):149-50.

5. Serrano-Cumplido A, Antón-Eguía Ortega PB, Ruiz García A, Olmo Quintana V, Segura Fragoso A, Barquilla Garcia A, et al. COVID-19. La historia se repite y seguimos tropezando con la misma piedra. SEMERGEN. 2020;46:48-54.

6. Vázquez Canales LM, Pujol Flores A. Los pilares de la Atención Primaria en tiempos de la COVID-19 [Internet]. AMF, Actualización de Medicina de Familia; 2020 [citado 6 de octubre de 2020]. Disponible en: https://amf-semfyc.com/web/article_ver.php?id=2663\&utm_content=buffere6b1f\&utm_medium=social\&utm source=twitter.com\&utm_campaign=buffer.

7. Aguilar-Barojas S. Fórmulas para el cálculo de la muestra en investigaciones de salud. Salud Tab. 2005;11:333-8.

8. Comisión Económica para América Latina y el Caribe. Salud y economía: una convergencia necesaria para enfrentar el $\mathrm{CO}$ VID-19y retomar la senda hacia el desarrollo sostenible en América Latina y el Caribe [Internet]. CEPAL, Comisión Económica para América Latina y el Caribe; 2020 [citado 24 de octubre de 2020]. Disponible en: https://www.cepal.org/es/publicaciones/45840-salud-economia-convergencia-necesaria-enfrentar-covid-19-retomar-la-senda.

9. The role of family physicians in a pandemic: A blueprint. [Internet]. ClinOwl; 2020 [citado 24 de octubre de 2020]. Disponible en: https://clinowl.com/the-role-of-family-physicians-in-a-pandemic-a-blueprint/.

10. Morens DM, Daszak P, Markel H, Taubenberger JK. Pandemic CO VID-19 Joins History's Pandemic Legion [Internet]. American Society for Microbiology, mBio; 30 de junio de 2020 [citado 6 de octubre de 2020]. Disponible en: https://mbio.asm.org/content/11/3/e00812-20.

11. Han E, Jin Tan MM, Turk E, Sridhar D, Leung GM, Shibuya K, et al. Lessons learnt from easing COVID-19 restrictions: an analysis of countries and regions in Asia Pacific and Europe. Lancet. 2020;396(10261):1525-34.
12. Lim WH, Wong WM. COVID-19: Notes from the Front Line, Sin gapore's primary health care perspective. Ann Fam Med. 2020;18(3):259-61.

13. Sarti TD, Lazarini WS, Fontenelle LF, Almeida APSC. What is the role of Primary Health Care in the COVID-19 pandemic? Epidemiol Serv Saude. 2020;29(2):e2020166.

14. Oseni TIA, Agbede RO, Fatusin BB, Odewale MA. The role of the family physician in the fight against Coronavirus disease 2019 in Nigeria. Afr J Prim Health Care Fam Med. 2020;12(1):e1-e3.

15. Lemire F, Slade $S$. Reflections on family practice and the pandemic first wave. Can Fam Physician. 2020;66(6):468.

16. Ayub M, Arshad D, Maqbool N, Zahid M, Malik RS, Rizvi ZA, et al. Physicians' attitudes towards treating patients in the context of COVID-19 pandemic in Pakistan. Cureus. 2020;12(9):e10331.

17. Ortiz Z, Antonietti L, Capriati A, Ramos S, Romero M, Mariani J, et al. Preocupaciones y demandas frente a COVID-19. Encuesta al personal de salud. MEDICINA (Buenos Aires). 2020;80(Supl. III):16-24.

18. Minué Lorenzo $S$, Bravo Toledo R, Simó Miñana J. Lecciones no aprendidas de la pandemia de la COVID-19 [Internet]. AMF, Actualización de Medicina de Familia; 2020 [citado 24 de octubre de 2020]. Disponible en: https://amf-semfyc.com/web/article_ ver.php?id=2717.

19. Lauer J, Kastner J, Nutsch A. Primary care physicians and pandemic influenza: an appraisal of the 1918 experience and an assessment of contemporary planning. J Public Health Manag Pract. 2008;14(4):379-86.

20. Yu EYT, Leung WLH, Wong SYS, Liu KSN, Wan EYF. How are family doctors serving the Hong Kong community during the $\mathrm{CO}$ VID-19 outbreak? A survey of HKCFP members. Hong Kong Med J. 2020;26:176-83.

21. Starfield B, Shi L, Macinko J. Contribution of primary care to health systems and health. Milbank Q. 2005;83(3):457-502.

22. León Martín AA. COVID 19, una nueva etapa de liderazgo perdido. Rev Clin Med Fam. 2020;13:114-5.

23. Pallarés Carratalá V, Górriz-Zambrano C, Llisterri Caro JL, Gorriz JL. La pandemia por la COVID-19: una oportunidad para cambiar la forma de atender a nuestros pacientes. Semergen. 2020;46(Suppl 1):3-5.

24. American Academy of Family Physicians. Telehealth and telemedicine [Internet]. American Academy of Family Physicians [citado 24 de octubre de 2020]. Disponible en: https://www.aafp. org/about/policies/all/telehealth-telemedicine.html.

25. Curioso WH, Galán-Rodas E. El rol de la telesalud en la lucha contra el COVID-19 y la evolución del marco normativo peruano. Acta Méd Perú. 2020;37(3):366-75.

26. Guía de Buenas Prácticas y Recomendaciones en Telemedicina durante epidemia de COVID-19 en Chile - CENS [Internet]. Chile: Centro Nacional en Sistemas de Información en Salud [citado 24 de octubre de 2020]. Disponible en: https://cens.cl/guia-buenas-practicas-telemedicina/.

27. Teleconsulta durante una pandemia [Internet]. Organización Panamericana de la Salud, Organización Mundial de la Salud [citado 24 de octubre de 2020]. Disponible en: https://iris.paho.org/ handle/10665.2/52007. 